\title{
DYNAMIQUE DES NOUVELLES TECHNOLOGIES RIZICOLES EN AGRICULTURE DE SUBSISTANCE : CAS DE LA RIZICULTURE BETE EN ZONE FORESTIERE DE CÔTE D'IVOIRE
}

\author{
S. DOUMBIA ${ }^{1}$, E. M. DEPIEU' ${ }^{1}$ et J.Z. KELI ${ }^{1}$ \\ ${ }^{1}$ Centre National de Recherche Agronomique (CNRA), 01 BP 1740 Abidjan 01, Côte d'Ivoire. \\ E-mail : moulouck2001@yahoo.fr
}

\begin{abstract}
RESUME
Une analyse rétrospective des modèles d'introduction et de diffusion des variétés améliorées de riz pluvial en Côte d'Ivoire a été effectuée. Plusieurs contraintes, qui empêchent l'adoption de ces nouvelles technologies ont été identifiées. La démarche est illustrée par les expériences menées depuis environs 30 ans, en zone forestière au Centre-Ouest de la Côte d'Ivoire. Plusieurs raisons expliquent ce faible taux d'adoption : D'une part, au plan macro-économique, l'option d'une politique de soutien à la consommation a amené les pouvoirs publics à compter sur les importations pour satisfaire la demande nationale. D'autre part, aux plans institutionnel et organisationnel, on assiste à un manque de coordination entre la recherche, le développement et les ONG dans le processus d'introduction et de diffusion des nouvelles variétés. Les vrais interlocuteurs en riziculture, que sont les femmes, ont été, pendant longtemps, négligées par ces structures. En plus, il y a eu un manque d'adéquation entre certains critères de sélection variétale et les besoins des producteurs. Ce qui a entraîné une inadaptation des nouvelles variétés au contexte traditionnel de la production. En définitive, malgré un environnement agro-écologique favorable à la production du riz, cette denrée constitue, malheureusement, une contrainte à la réalisation d'une politique nationale de sécurité alimentaire. Les auteurs, tirant les leçons des expériences passées formulent quelques recommandations qui devraient favoriser une meilleure introduction et une diffusion, plus large, des nouvelles variétés de riz NERICA (New Rice for Africa).
\end{abstract}

Mots clés : Riz, modèle de diffusion, variétés améliorées, zone forestière, Côte d'Ivoire.

\begin{abstract}
NEW RICE AGRICULTURAL TECHNOLOGY DYNAMICS IN SUBSISTENCE AGRICULTURE : A CASE STUDY THROUGH BETE RICE FARMING IN THE FOREST ZONE OF CÔTE D'IVOIRE
\end{abstract}

A retrospective analysis was undertaken on the introduction and spread models of new improved rain fed rice varieties in Côte d'lvoire. Several constraints stand on the way to the adoption of these farming technologies. The approach is illustrated through experiments undertaken for nearly 30 years in Gagnoa and Saïoua areas, located in the forest zone in Centre-West Côte d'Ivoire. Several raisons can explain the poor adoption rate by the population of new rice varieties: first at the macroeconomic level, we notice that government officials have adopted a deliberate low purchase price policy for the local rice product, relying on importations to satisfy the rice demand at national level. Moreover, there is a lack of coordination between research, development organizations and NGOs in both the introduction and the diffusion processes of new rice varieties. Women, who are at the forefront of rice cultivation have been, for long, neglected. Moreover, there a lack of adequacy between some selection criteria of new rice varieties and the needs of producers, which led to a failure to adjust new rice varieties to the traditional context of production. Finally, despite the favourable agro ecological environment to rice production, this foodstuff constitutes unfortunately a constraint that stands in the way to the achievement of national food security policy. The authors, learning from the past experiences, give some recommendations, which should allow for a better introduction and a large diffusion of new rice varieties, such as NERICA (New Rice for Africa).

Keywords : Rice, diffusion model, improved varieties, forest zone, Côte d'lvoire. 


\section{INTRODUCTION}

La situation actuelle de la riziculture dans les pays de l'Union économique et monétaire ouest africaine (UEMOA), de manière générale, et de la Côte d'Ivoire, en particulier, est caractérisée par un déficit constant entre le niveau de la production et celui de la consommation. Cette tendance trouve en partie sa source dans le décalage qui existe entre les prix du riz sur le marché mondial et ceux beaucoup plus élevés auxquels aboutissent les filières nationales de production (Djéa, 2003). D'après la FAO (1994), les gouvernements ont utilisé cette marge entre le prix du riz importé et celui du riz produit localement pour tenter de concilier deux objectifs à priori contradictoires, à savoir maintenir un prix du riz acceptable pour les consommateurs urbains et favoriser le développement d'une riziculture nationale généralement encore peu performante et aux coûts de revient élevés. Cette contradiction est d'autant plus difficile à gérer que la part du riz dans la consommation nationale est importante. Ce constat s'est doublé depuis la dévaluation, d'une nouvelle contradiction. En effet, toujours selon la FAO (1994), la question fondamentale qui se pose désormais est de savoir comment accroître le disponible national en riz alors que la dévaluation pénalise la plupart des itinéraires d'intensification ? Par rapport à cette interrogation, la variété constitue une première réponse de la part de la recherche. En effet, la variété apparaît comme une technologie agricole intéressante en soi, en ce sens que son adoption, peu risquée d'un point de vue financier constitue une source potentielle de productivité élevée (Poisson et Doumbia, 1987). C'est d'ailleurs pourquoi la semence représente presque toujours le premier thème diffusé par la recherche en attendant la définition plus globale des conditions de l'intensification agricole et de la stabilisation des systèmes de culture à base de riz. Ce constat a conduit très tôt la recherche à mettre l'accent sur la création et l'amélioration variétale (Chabrolin, 1969). Les résultats issus de ces travaux ont été au fur et à mesure proposés à l'adoption aux paysans depuis la fin des années 1960. Trente ans après, quel bilan est-on en mesure de présenter ? C'est dans cette perspective que s'inscrit notre étude dont l'objectif général est de réaliser le bilan des introductions de variétés améliorées de riz en milieu rural pendant cette période. De façon spécifique, il s'agit (i) d'analyser les modèles d'introduction et de diffusion des variétés améliorées de riz pluvial inscrites dans le Catalogue officiel (MINADER, 2002), (ii) de caractériser le contexte de leur production, (iii) d'évaluer le taux d'adoption de ces variétés et enfin (iiii) d'identifier les contraintes qui empêchent l'adoption de ces nouvelles technologies.

\section{MATERIEL ET METHODES}

L'étude s'est déroulée en zone forestière dans le Centre-Ouest de la Côte d'Ivoire au cours des campagnes agricoles 2003 et 2004. Elle a couvert les villages de Nékéidé, Zahibohio et Mahiboua dans la sous-préfecture de Gagnoa ; Balam, Godoua, Ouandahio et Digbam dans la sous-préfecture de Saïoua. Toute cette région appartient à l'aire socioculturelle Krou, originellement de peuplement bété, avec cependant des poches importantes de populations allogènes et allochtones, notamment, dans les villes. Le travail a reposé, d'une part, sur la recherche documentaire et, d'autre part, sur la méthode accélérée de recherche et de planification participative (MARP) avec utilisation d'interview semi structurée comme méthode d'investigation. L'interview de groupe a concerné 71 et 68 rizicultrices respectivement à Gagnoa et à Saïoua. Les rendements ont été estimés à la récolte à partir des carrés de rendement répartis sur un échantillon de 50 rizières, les mesures de superficies ont été réalisées avec le «Global Positioning System» (GPS).

\section{RESULTATS}

\section{LE CONTEXTE DE LA PRODUCTION DU RIZ EN ZONE FORESTIERE DE CÔTE D'IVOIRE}

La zone forestière se caractérise depuis l'indépendance du pays en 1960 par un flux migratoire important dû à un potentiel agroécologique élevé. Ce fait a créé une menace permanente sur les ressources forestières et les équilibres écologiques et s'est traduit par la quasi-disparition de la forêt primaire et une perturbation importante du régime pluviométrique. En effet, l'analyse de la pluviosité de 1941 à 2000 indique une tendance décroissante. La moyenne stabilisée y est de $1500 \mathrm{~mm}$ pour la période 1941 - 1970 et de $1350 \mathrm{~mm}$ pour la période 1971 - 2000, soit une baisse de 
$150 \mathrm{~mm}$. La conjonction de cet ensemble d'évolutions défavorables fait désormais de la définition des conditions techniques et sociales de mise en place des systèmes d'exploitation du milieu durable, un des enjeux du développement agricole et de la recherche dans cette zone.

Dans la région qui nous intéresse, la culture du riz est importante et demeure le fait de petits producteurs, des femmes essentiellement. Elle est pratiquée sur des superficies modestes, inférieures en moyenne à 1 ha et semées en plusieurs variétés (Doumbia, 2003). Avec plus des deux tiers de l'effectif ayant moins de 45 ans, les agricultrices que nous avons interviewées peuvent-être considérées comme jeunes. Ces femmes sont toutes mariées, le mariage leur offrant habituellement une plus grande opportunité d'accès à la terre. Le capital d'exploitation n'est jamais important, et se limite à quelques outils dont la machette et la daba. Le travail essentiellement d'origine familiale et limité représente le principal facteur de production avec la terre. On dénombre en moyenne deux à trois actifs agricoles par exploitation. Le niveau des techniques culturales est rudimentaire. Le travail du sol est superficiel, le semis en ligne est exceptionnel. II est au contraire, pratiqué le semis en poquets non alignés, qui se traduit par une faible densité à la levée. L'utilisation des intrants agricoles est rare, sauf lorsque ceux-ci sont subventionnés dans le cadre de projets de développement. Le volume total de la production ainsi obtenue est faible et concourt à marquer fortement le caractère de subsistance de l'activité agricole. Nos résultats d'enquête sur le suivi des stocks de riz dans la région de Saïoua en 2004 ont montré que seulement trois mois après la récolte qui intervient de juin à septembre, plus de $50 \%$ des ménages agricoles procèdent à des achats de riz. La soudure pour certains ménages commence déjà en février pour s'installer confortablement et de manière quasi générale en mars-avril.

\section{ANALYSE DE L'ADEQUATION ENTRE L'OFFRE ET LA DEMANDE DE VARIETES NOUVELLES DE RIZ}

\section{L'offre de la recherche en matière de variétés de riz pluvial}

Dans les pays d'Afrique francophone, l'Institut de recherche des cultures vivrières en agronomie tropicale (IRAT) a joué dans le domaine de l'amélioration et de la création variétale un rôle de pionnier. À partir de 1969, les programmes de recherche ont été orientés dans les deux directions suivantes (Chabrolin, 1969). D'une part, l'amélioration du milieu, avec des études axées sur la fertilisation, l'amélioration des pratiques culturales, l'élimination des parasites et, d'autre part, l'amélioration des plantes. $\mathrm{Ce}$ second axe avait pour objectif l'adaptation du cycle végétatif aux exigences du milieu ou de l'utilisateur, l'augmentation de la productivité, la résistance aux maladies, à l'égrenage et à la saliure le cas échéant. Un accent particulier a été mis sur la création de variétés d'une architecture améliorée, de petite taille à feuille érigées, très résistantes à la verse et ne réagissant pas à la fumure azotée par une exubérance exagérée de leur appareil végétatif. Les travaux ont porté également sur les aspects technologiques (grain blanc de format long ou demi long, translucide, riz brisant peu à l'usinage). D'une manière générale, ces variétés n'expriment pleinement leurs potentialités que lorsque les conditions de culture sont améliorées (fertilisation, lutte contre les mauvaises herbes). Un grand nombre de variétés ont ainsi été créées par rapport à ces orientations dans le cadre de I'IRAT et de l'IDESSA. Une synthèse réalisée par Poisson et Doumbia (1987) en dresse le tableau suivant. Ces variétés selon ces deux auteurs sont résistantes aux maladies et en particulier à la pyriculariose, et tolérantes aux périodes de sécheresse (IRAT 104, IRAT 170) si fréquentes dans certaines régions de Côte d'Ivoire. Leur taille est plus courte que celle des variétés traditionnelles afin de les rendre plus résistantes à la verse et faciliter leur récolte mécanique, en autorisant une meilleure réponse à la fumure en condition de culture intensive. D'autres caractères comme le trop fort égrenage à maturité ont été améliorés (IRAT 112, IDSA 6). La pilosité des grains jugée irritante par l'agriculteur a été supprimée (IRAT 170, IDSA 6). Un type nouveau de plante a été obtenu avec IDSA 6 pour s'accommoder aussi bien aux conditions pluviales qu'inondées. La qualité du grain n'a pas été laissée pour compte non plus. Les connaisseurs retrouveront dans IRAT 136 le goût de «Morobérékan», dans IRAT 112 la translucidité et le goût de "Dourado», et dans IDSA 6 au grain très fin, parfaitement translucide, une bonne tenue à la cuisson et une saveur agréable. La précocité qui autorise une plus grande latitude quant à la période de semis a été recherchée à travers les variétés (IRAT 112 et IRAT 144), tandis que les variétés (IRAT 104, 
IRAT 136, IRAT 170, et IDSA 6) de cycle moyen s'adaptent bien à des semis à date optimale. Cet ensemble de variétés améliorées (anciennes et récentes) recensées dans le Catalogue officiel des variétés de riz (MINADER, 2002) constitue l'offre en variétés pluviales de riz de la recherche en Côte d'Ivoire.

\section{Quelques caractéristiques de la demande paysanne en matière de variétés de riz}

L'essentiel du stock local de variétés traditionnelles de riz est le fruit d'un long processus empirique d'évaluation par les agricultrices. Ces variétés sont de cycle moyen à long, passablement résistantes à la sécheresse. Ce sont des variétés de grande taille (environ $1,20 \mathrm{~m}$ à maturité) afin de permettre aux rizicultrices de procéder à la récolte manuelle sans trop de peine ; elles égrènent peu, ce qui constitue un réel atout dans un milieu de production où le paddy fait l'objet de nombreuses manipulations depuis la récolte jusqu'à l'entreposage dans les greniers. En années pluviométriques normales et malgré les conditions extensives de culture, les rendements sont bas mais stables d'une année à l'autre. Ils sont même comparables à ceux des variétés améliorées dans les conditions paysannes de culture. En effet nos estimations dans la région de Gagnoa indiquent des rendements moyens respectifs de 1,975 tonnes/ha (CV $=41 \%$ ) et $1,606$ tonnes/ha ( $C V=37 \%)$ pour les variétés améliorées et pour les variétés traditionnelles. Ces dernières sont créditées, par ailleurs, d'un goût excellent par rapport aux critères locaux d'appréciation. Toutefois, depuis une vingtaine d'années environ, avec la tendance baissière de la pluviométrie, les critères de résistance à la sécheresse et la précocité connaissent un regain d'intérêt, tandis que l'enthousiasme pour les caractéristiques organoleptiques, sans décroître, ne paraît cependant plus aussi déterminant dans le choix d'une variété. Dans notre zone d'étude, la demande locale en matière de variété de riz pluvial porte surtout sur des variétés résistantes à la sécheresse et/ou à vocation mixte (pouvant être cultivé sur le plateau comme en bas de pente), productives et précoces. Toute variété candidate à l'adoption devra également être de grande taille, présenter une bonne résistance à l'égrenage et avoir éventuellement un bon goût. La capacité à croître et à se développer sur des sols peu fertiles constituerait un atout indéniable.

INTRODUCTIONDES VARIETES AMELIOREES DE RIZ EN MILIEU PAYSAN

\section{Les modèles d'introduction et de diffusion des variétés nouvelles de riz en zone forestière de Côte d'Ivoire}

L'activité d'introduction et de diffusion des nouvelles variétés de riz a pendant longtemps été une préoccupation de la recherche seule. Cette institution s'en est acquittée dans un premier temps avec des moyens limités, les équipes étant réduites dans la plupart des cas à un seul chercheur. Dans un second temps, on a assisté à une amorce de collaboration entre la recherche et les structures d'encadrement. En zone forestière, le tandem IRAT/SATMACl a connu un début de fonctionnement au début des années 1980. Seul le Projet Agricole du Centre Ouest (PACO) a tenté de formaliser une telle collaboration en créant en son sein une cellule de Recherche/développement animée à la fois par un chercheur et des agents du développement. Par la suite, la collaboration interinstitutionnelle s'est relâchée, en devenant sporadique. Parallèlement à ce relâchement, on a assisté à l'irruption dans le champ d'activité de l'introduction et de la diffusion des nouvelles variétés de riz, de nouveaux intervenants. Parmi ceux-ci, on note des personnes agissant à titre individuel (agents de la SATMACI), des organisations non gouvernementales (ONG) telles que «Doubéhi» International et la Communauté laïque des missionnaires catholiques (CLMC), de même que des organisations internationales comme le Programme Alimentaire Mondial (PAM). Ces divers intervenants agissent pour des motifs les plus variés, sans coordination de leurs actions respectives. Ces structures procédaient ainsi aux introductions selon une approche descendante sans consultation préalable des bénéficiaires, faisant du coup l'impasse sur 
l'identification et la caractérisation de la demande sociale locale en matière de nouvelles technologies. Par ailleurs, rarement ces interventions se sont inscrites dans la durée, avec pour conséquence une introduction intempestive et inadaptée des variétés de riz. Rarement aussi, des bilans ont été établis, aucune donnée fiable n'existe pour ces actions en ce qui concerne les taux d'adoption. Cependant, depuis le milieu des années 1990, on assiste à un effort d'organisation et de rationalisation des actions d'introduction et de diffusion des variétés améliorées de riz. A l'avant garde de ce renouveau se trouvent des ONG et les structures de recherche travaillant en partenariat. Ce partenariat est caractérisé par une approche participative, des interventions coordonnées avec en toile de fonds le souci d'une implication effective des femmes. Dans le cas de l'ONG, Organisation des volontaires pour le développement local (OVDL) à Saïoua, ce partenariat concerne les interventions suivantes : le Projet d'appui à l'adoption et à la diffusion des variétés dans les systèmes à base de riz (PADS) et le Projet de sélection variétale participative (PVS). Ces deux projets ont été exécutés pour le compte de l'ADRAO. On note aussi le Projet communautaire de base de production de semence (CBSS) mis en oeuvre pour le compte du Ministère de l'Agriculture et le Projet "Riz pour tous" exécuté pour le compte du Programme National Riz (PNR). En prélude à ces interventions, on a remarqué un réel effort de structuration du milieu rural. La constitution de nombreux groupements de femmes rizicultrices en zone forestière constitue, aujourd'hui, un des résultats les plus tangibles de ces mutations. Pour la seule année 2004, on a dénombré dans la région de Saïoua selon I'OVDL, la création de 65 associations regroupant 1625 membres répartis au niveau de 34 villages. Ces groupements sont crées à partir de critères simples, sur la base du volontariat avec un mode de fonctionnement souple. La production et la commercialisation des semences constituent l'activité principale du programme d'action de ces groupements dont les membres reçoivent un minimum de formation aux techniques culturales. De manière générale, la demande en semence excède l'offre et les statistiques de vente montent à 2,19 tonnes et 15,82 tonnes de semence respectivement pour les années 2003 et 2004 . Quoique le marché des semences soit un marché étroit, les semences vendues entre 200 et 400 FCFA $/ k g$, constituent pour l'heure une excellente opportunité de commercialisation pour les groupements, en comparaison du paddy vendu à $90 \mathrm{FCFA} / \mathrm{kg}$ bord champ. Sur le terrain, chaque groupement dispose d'une parcelle communautaire d'un hectare à laquelle tous les membres consacrent une journée de travail par semaine. Par ailleurs, de part leur mode de fonctionnement, les groupements constituent un relais efficace dans l'introduction et la diffusion des nouvelles variétés. Ce processus repose sur les quatre étapes suivantes: (i) chaque groupement reçoit à sa création de la semence d'une ou deux variétés nouvelles de riz, (ii) le groupement est tenu après la récolte de céder à chaque membre une certaine quantité de la production sous forme de semence, (iii) le bénéficiaire est à son tour invité à ensemencer une parcelle lui appartenant en propre avec la semence obtenue. Ce processus est connu sous le nom de "Opération une femme, une parcelle", (iiii) à partir de cette étape se crée une dynamique de diffusion de la variété qui prend appui sur divers événements de la vie courante qui constituent autant d'opportunités d'échange et de diffusion du matériel végétal (voyages, funérailles, mariages, etc.) tant au sein d'un même village que d'un village à un autre. Plus généralement, une variété donnée diffuse dans le milieu "paysan" suivant les quatre modalités que sont le don, le paiement en nature contre la réalisation d'un travail, l'achat et l'échange. Le don de semence peut être motivé par l'existence de lien de parenté ou du simple fait de l'amitié ou du voisinage. Le cas de rétribution a lieu lors de la récolte où les personnes venues vous aider reçoivent en contrepartie deux à trois bottes de paddy pour chaque lot de dix bottes de paddy récoltées. L'achat de semence, lorsqu'il a lieu n'échappe pas à la loi de l'offre et de la demande. Les prix de la semence étant le plus élevé juste avant la période des semailles tandis que l'échange de semences se produit conformément aux règles du troc.

\section{Les variétés introduites et diffusées en zone forestière}

L'introduction des nouvelles variétés de riz dans la région de Gagnoa s'est faite par vagues successives d'environ une dizaine d'années chacune, au cours de notre période de référence qui va de la fin des années 1960 au début des années 2000 (Tableau 1). La décennie 1980 1990 a constitué une période charnière. En effet, au niveau du matériel végétal, les variétés 
introduites en Côte d'Ivoire et adaptées à différentes régions du pays comme «Morobérékan», "Dourado précoce» et «IGUAPE Cateto» ont progressivement été remplacées d'abord par des variétés créées dans le cadre de l'IRAT-Côte d'Ivoire, et ensuite par des variétés créées dans le cadre de l'Institut des Savanes (IDESSA), structure née au milieu de la décennie de référence. La troisième et dernière décennie a vu au plan du matériel végétal l'arrivée des variétés de type WAB sous l'impulsion de L'ADRAO, structure sous régionale de recherche. Les NERICA constituent les toutes dernières créations de la part de cet organisme.
Au niveau de la région de Saïoua, les dernières variétés introduites sont le fait de l'OVDL dont l'expérience dans la région remonte seulement à 1996. Depuis cette date, trois vagues assez distinctes de variétés ont été introduites comme le montre le tableau 2. Pour la période allant de 1996 à 2000, grâce à la collaboration d'une ONG (Winrock International) et de la Recherche (ADRAO, IDESSA, CNRA), I'OVDL a procéder à l'introduction d'un peu moins d'une dizaine de variétés nouvelles de riz. De 2000 à 2003, grâce essentiellement à la collaboration de l'ADRAO, les variétés NERICA ont été introduites dans la région, et depuis 2003 sous l'impulsion du PNR, les variétés WITA9 et Pomagbou ont été introduites.

Tableau 1 : Evaluation dans le temps des introductions de variétés de riz pluvial en zone forestière de Côte d'Ivoire

Evaluation of rainfed rice introduction at different periods of time in the forest zone of Côte d'Ivoire.

\begin{tabular}{|c|c|c|c|c|}
\hline $\begin{array}{l}\text { Période } \\
\text { d'introduction }\end{array}$ & Origine de la variété & Variétés introduites & $\begin{array}{c}\text { Présence de la variété } \\
\text { au champ en } 2003\end{array}$ & $\begin{array}{l}\text { Village } \\
\text { concerné }\end{array}$ \\
\hline $1968 / 1969$ & Agent SATMACI & $\begin{array}{c}\text { Jaya } \\
\text { Morobérékan }\end{array}$ & $\begin{array}{l}\text { Non } \\
\text { Non }\end{array}$ & Zahibohio \\
\hline 1970 & IRAT & SATMACI & Non & Mahiboua \\
\hline 1978 & SATMACI & Indéterminée & Oui & Zahibohio \\
\hline 1980 & IRAT & Chinois & Oui & Zahibohio \\
\hline 1985 & SATMACI & $\begin{array}{l}2 \text { variétés } \\
\text { indéterminées }\end{array}$ & Non & Mahiboua \\
\hline $1985 / 1986$ & IRAT & $\begin{array}{l}\text { IRAT } 110 \\
\text { IRAT } 112\end{array}$ & $\begin{array}{l}\text { Non } \\
\text { Non }\end{array}$ & $\begin{array}{l}\text { Nékéidé et } \\
\text { Zahibohio }\end{array}$ \\
\hline $1988 / 1990$ & $\begin{array}{l}\text { IDESSA } \\
\text { IDESSA } \\
\text { IDESSA } \\
\text { ADRAO }\end{array}$ & $\begin{array}{c}\text { IDSA } 6 \\
\text { IDSA } 10 \\
\text { IAC } 165 \\
\text { DR } 2\end{array}$ & $\begin{array}{l}\text { Non } \\
\text { Non } \\
\text { Oui } \\
\text { Oui }\end{array}$ & Mahiboua \\
\hline $1994 / 1997$ & CLMC & $\begin{array}{c}\text { IAC } 165 \\
\text { IDSA } 10 \\
\text { WAB 56-50 } \\
\text { IGUAPE Cateto }\end{array}$ & $\begin{array}{c}\text { Oui } \\
\text { Non } \\
? \\
\text { Non }\end{array}$ & Tiégbahi \\
\hline 1996 & PAM & $\begin{array}{c}1 \text { Variété } \\
\text { Indéterminée }\end{array}$ & Non & Zahibohio \\
\hline 2002 & Privé & NERICA 1 & Oui & Nékéidé \\
\hline 2003 & $\begin{array}{l}\text { ONG Doubehi } \\
\text { International }\end{array}$ & NERICA 1 & Oui & $\begin{array}{l}\text { Zahibohio } \\
\text { Mahiboua }\end{array}$ \\
\hline
\end{tabular}


Tableau 2 : Liste nominative des variétés introduites depuis 1996 et des variétés actuellement cultivées par les paysans dans la région de Saïoua

Introduced varieties since 1996 and those actually cultivated by farmers in the region of Saïoua, forest zone of Côte d'Ivoire.

\begin{tabular}{lcc}
\hline & \multicolumn{2}{c}{ Variétés } \\
\cline { 2 - 3 } Période & Variétés introduites & $\begin{array}{c}\text { Variétés actuellement } \\
\text { cultivées par les paysans }\end{array}$ \\
\hline \multirow{2}{*}{$1996-2000$} & IDESSA 77, IDESSA 78 & WAB 5650, IDESSA 78 \\
& IDESSA 85, WAB 5650 & IDESSA 85, Palawan. \\
& WAB 56125, WAB 56104 & WAB 638-1 \\
$2000-2003$ & WAB 638-1, Palawan & Nerica1 (BONFANI) \\
Depuis 2003 & Nerica1 & Nerica2 (Keah) \\
\hline
\end{tabular}

\section{Evaluation du taux d'adoption des nouvelles variétés de riz.}

Notre évaluation repose sur l'appréciation de (i) la superficie moyenne par rizière, (ii) la superficie emblavée par rizière en variétés améliorées et (iii) le ratio de la superficie en variétés améliorées à la superficie totale en riz par rizière. Le premier paramètre nous informe quant à la distribution régionale de la superficie moyenne par rizière, tandis que les deux derniers constituent de bons indicateurs du taux d'adoption des variétés améliorées. La distribution par classe de superficie exprimée en hectares des parcelles de riz pluvial dans la région de Saïoua apparaît au tableau 3. Cette distribution se caractérise par une superficie extrêmement faible des rizières : $95 \%$ d'entre elles mesurent au plus 1,5 ha tandis que seuls $5 \%$ des rizières ont une superficie supérieure ou égale à 1,75 ha.

Les résultats relatifs au ratio de la superficie en variétés améliorées de riz à la superficie totale en riz par rizière apparaissent au tableau 4. La moyenne régionale du taux d'adoption s'élève à $14 \%$. Cette moyenne cache des disparités importantes tant d'un village à un autre qu'au sein d'un même village. De même, on note une très grande variabilité du niveau d'adoption au sein de chacun des villages. En effet, le taux d'adoption varie de $4 \%$ à «Ouandahio» à $21 \%$ à "Godoua». Cette grande variabilité des résultats est attestée par les valeurs élevées du coefficient de variation (CV). Ce chiffre varie d'environ $75 \%$ à «Digbam» à environ $120 \%$ au niveau régional. En valeur absolue, le taux d'adoption moyen de $14 \%$ est faible et suggère l'existence de nombreuses contraintes à la diffusion des nouvelles variétés de riz dans la région. Les valeurs élevées des coefficients de variation que l'on constate indiquent en partie que les agricultrices ne sont pas au même niveau d'information par rapport à l'innovation, ni d'un village à un autre, ni au sein d'un même village. En outre comme il apparaît au tableau 1, certaines variétés sont classées soit comme indéterminée ou soit portant le nom de la structure qui pour la première fois les a introduites au village. Il est, parfois, fait allusion à l'origine géographique de la variété. Ce phénomène qui consiste de la part des rizicultrices à rebaptiser une variété donnée de riz est assez courant en milieu villageois et en ajoute à la confusion au cours des évaluations, contribuant ainsi à sous estimer le taux réel d'adoption d'une variété donnée. En définitive dans notre zone d'étude, si l'on fait abstraction des variétés NERICA d'introduction récente, seules deux variétés (IAC 165 et DR2) sur dix identifiées continuent d'être exploitées par les agricultrices dans la région de Gagnoa. Dans la région de Saïoua où l'activité d'introduction date seulement de 1996, six variétés sur dix sont encore cultivées. 
Tableau 3 : Distribution par classe de superficie (ha) des parcelles en riz pluvial de la région de Saïoua.

Rice area distribution by class of magnitude in the region of Saïoua, forest zone of Côte d'Ivoire.

\begin{tabular}{lccc}
\hline $\begin{array}{l}\text { Classe de superficie } \\
\text { (ha) }\end{array}$ & $\begin{array}{c}\text { Effectif } \\
\text { par classe (n) }\end{array}$ & $\begin{array}{c}\text { Pourcentage } \\
\text { par classe } \\
(\%)\end{array}$ & $\begin{array}{c}\text { Pourcentage } \\
\text { cumulé (\%) }\end{array}$ \\
\hline 0,09 à 1 & 48 & 73,85 & 73,85 \\
1 à 1,5 & 14 & 21,54 & 95,39 \\
1,5 à 1,75 & 3 & 4,61 & 100 \\
\hline
\end{tabular}

Tableau 4 : Ratio superficie en variétés de riz améliorées sur superficie totale en riz. dans la région de Saïoua.

Ratio distribution of improved rice area to total rainfed rice area in the region of Saïoua, forest zone of Côte d'Ivoire.

\begin{tabular}{lccccc}
\hline \multirow{2}{*}{ Désignation } & S/Préfecture & \multicolumn{4}{c}{ Villages } \\
\cline { 3 - 6 } & Saïoua & Balam & Godoua & Ouandahio & Digbam \\
\hline Moyenne & 0,14 & 0,14 & 0,21 & 0,04 & 0,19 \\
$\begin{array}{l}\text { Coefficient de } \\
\text { variation (\%) }\end{array}$ & 119,78 & 73,44 & 111,54 & 143,93 & 77,84 \\
\hline
\end{tabular}

\section{Les contraintes à l'adoption de nouvelles variétés de riz}

Nous avons établi une grille de critères justifiant l'abandon d'une variété donnée de même que les défauts relatifs à chacune des variétés mises en cause. En ce qui concerne les causes agronomiques, la taille courte et le fort taux d'égrenage constituent les causes les plus souvent citées. Ces causes ont été suffisantes aux yeux des rizicultrices pour justifier l'abandon des variétés IDSA 10 (petite taille) et la variété indéterminée SATMACI1 (fort taux d'égrenage). Au niveau des causes technologiques, la mauvaise tenue à la cuisson et le fait que pendant le décorticage, les grains s'écrasent au pilage pour donner de la farine ont constitué pour les rizicultrices des motifs suffisants d'abandon des variétés IDSA10, IRAT110, IRAT112 et SATMACI2. Les variétés IDSA10 et IDSA46 ont été abandonnées à Saïoua pour des raisons similaires, tandis que la variété IDSA85 sans être abandonnée souffre de sa réputation de variété à fort taux d'égrenage. II est peut-être trop tôt pour porter un jugement définitif, mais les variétés NERICA, appréciées par ailleurs pour leur précocité, leur résistance à la sécheresse et leur productivité, rallient peu le suffrage des rizicultrices pour ce qui concerne le goût et la taille.

\section{DISCUSSION}

Notre analyse des modèles d'introduction et de diffusion des nouvelles variétés de riz indique que des changements importants, quoique récents sont intervenus dans ce domaine. Au départ, basées sur des approches descendantes et peu coordonnées, les actions d'introduction et de diffusion des variétés ont progressivement évolué vers des approches plus participatives dans le cadre d'un partenariat concerté entre la recherche, le développement et les ONG. Parallèlement à cette évolution, est intervenue la reconnaissance de la femme comme une interlocutrice privilégiée dans le processus de production des vivriers. De même, la création d'activités génératrices de revenus, par la possibilité de production et de commercialisation des semences par les femmes, en accompagnement de l'activité de dissémination des nouvelles variétés, constitue une autre caractéristique remarquable des évolutions récentes. Si la conjonction de ces différentes mutations favorables s'est traduite par une meilleure organisation du processus d'introduction et de diffusion des variétés nouvelles, les taux d'adoption constatés sur le terrain laissent cependant encore à désirer. Ces résultats mitigés tiennent à plusieurs raisons. 
En effet, malgré la reconnaissance de son statut de productrice, la femme est encore loin de détenir les principaux leviers de commande pour accomplir de manière efficace l'activité de production. En témoigne la faiblesse des superficies rizicultivées et le volume total de la production vivrière (Doumbia, 2003). En effet, la femme n'a accès à la terre qu'indirectement, c'est-à-dire à travers le mariage et accessoirement par héritage. Par ailleurs, la faiblesse des moyens financiers dont elle dispose est telle qu'elle ne peut effectuer des investissements qu'à minima dans l'activité de production. Tout au plus est-elle en mesure de lever pour la réalisation de travaux contraignants quelques journées de main d'œuvre salariée! Cette précarité de la condition de la femme déteint sur le volume final de la production de même qu'elle contraint l'adoption des innovations technologiques. En outre, l'orientation très marquée de la production agricole vers l'autoconsommation contribue à réduire significativement le surplus commercialisable et donc les revenus agricoles. En effet, selon les données de la Compagnie Ivoirienne de Développement des Vivriers (CIDV) relative à la campagne 1993 - 1994, près de $57 \%$ de la production locale à servi à l'autoconsommation, le surplus commercialisable représentant l'offre du secteur s'est établi à $43 \%$ de la production (MINAGRA, 1991). Les bas prix d'achat au producteur constituent pour beaucoup d'analystes un autre facteur responsable de la modicité du volume de la production vivrière, et partant les raisons du faible recours aux nouvelles technologies agricoles. De ce fait, nombre d'analystes plaident pour un relèvement de ces prix qui agissent comme des déterminants du revenu réel des consommateurs, et induisent au moins à court terme une augmentation de la production alimentaire. Toutefois, cette situation impose aux consommateurs à faible revenu un coût élevé à supporter. C'est la contradiction du "dilemme du prix de la nourriture" (Weber et al., 1988). Ce dilemme constitue la pierre angulaire de toute analyse de politique alimentaire. Un autre aspect du "dilemme du prix de la nourriture" a trait à la magnitude de la réponse de l'offre de produit à des prix d'achat élevés. L'orientation marquée de la production agricole vers la subsistance a amené certains analystes dont Badouin (1971) à conclure que la réponse de l'offre à une augmentation des prix des produits agricoles serait limitée. Scandizzo et Bruce (1980) ont montré qu'à long terme l'élasticité de l'offre estimée pour un ensemble de produits alimentaires de base au niveau de 103 pays en développement était inférieure à 0,5 dans $62 \%$ des cas et était négative dans $27 \%$ des cas. Une étude de simulation réalisée par Martin (1988) a clairement montré une réponse limitée de l'offre en céréales à une augmentation des prix d'achat au Sénégal. Cet auteur a conclu que même dans l'hypothèse d'une augmentation de $100 \%$ du prix d'achat des céréales, le degré d'autosuffisance alimentaire passerait de $47 \%$ à seulement $55 \%$, ce qui confirme la faiblesse de la réponse de l'offre de produit à des augmentations de prix. Les données de la littérature disponibles sur ce sujet en Côte d'Ivoire infirment dans une large mesure ces conclusions. En effet, d'après MINAGRA, 1991, le relèvement du prix d'achat officiel du paddy de 60 à 80 F CFA entre 1983 et 1985 a eu pour effet une nette reprise des niveaux de production d'environ $43 \%$. Les travaux de Koné (1993) confirment cette importance du prix d'achat au producteur. Cet auteur a montré dans une étude de l'impact de la vulgarisation sur l'efficacité économique des paysans dans le Nord-Ouest et l'Ouest de la Côte d'Ivoire que l'élasticité du prix de l'offre est de 0,79. Avant lui, Hirsch et al. (1984) avait abouti à des conclusions similaires toujours en Côte d'Ivoire dans le cadre d'une étude diagnostique de la filière riz.

\section{CONCLUSION}

Le bilan de l'introduction et de la diffusion des variétés améliorées de riz que nous avons établi s'est avéré riche d'enseignements. Il a confirmé, en précisant bien des aspects, l'inadéquation de nombre de critères de sélection variétale de la recherche au contexte traditionnel de la production de riz. En effet d'un côté, on note que la recherche s'efforce depuis plusieurs décennies à mettre au point des variétés pour des milieux améliorés dans le cadre d'une riziculture intensive, de l'autre les systèmes locaux de culture à base de riz demeurent désespérément extensifs avec une option marquée pour l'utilisation de variétés rustiques et adaptées au contexte traditionnel de production. Ce déphasage entre d'une part les objectifs de sélection de la recherche, et d'autre part les objectifs de production des riziculteurs explique en partie la faiblesse des taux d'adoption des variétés améliorées de riz. Toutefois, les analyses récentes ont permis de révéler l'existence d'un certains nombre de mutations 
en cours. Certaines sont favorables et d'autres, au contraire défavorables. Parmi les évolutions bénéfiques, on note l'amélioration du partenariat entre les divers intervenants dans le processus d'introduction et de diffusion des variétés améliorées de même qu'une meilleure organisation des rizicultrices. A l'opposé, la précarité de la condition de la femme en tant que productrice et la pratique d'une politique de soutien à la consommation méritent d'être indexées. Seule une prise en compte effective de ces contraintes par la définition d'un prix d'achat rémunérateur, véritable stimulant de la production dans le contexte ivoirien, et le renforcement de la condition de la femme en tant que productrice par une plus grande facilité d'accès aux facteurs de production et au crédit sont en mesure d'améliorer le taux d'adoption des nouvelles technologies rizicoles en milieu paysan.

\section{REFERENCES}

Badouin R. 1971. Economie rurale. Collection U, série " Sciences économiques et gestion ". Armand Colin éditeur, 1971, 598 p.

Chabrolin R. 1969. Les recherches rizicoles en Afrique Tropicale francophone et à Madagascar. Agronomie Tropicale 24 (1) : $15-38$.

Djea Y. F. A. 2003. Estimation des fonctions d'offre et de demande de riz en Côte d'Ivoire (19602000). Mémoire de fin d'études pour l'obtention du diplôme d'agronomie approfondie, option agroéconomie.

Doumbia S. 2003. Trente années d'introduction et de diffusion de variétés de riz pluvial par la recherche en zone forestière de Côte d'Ivoire : Bilan et perspectives. Centre National de Recherche Agronomique (CNRA) / Direction régionale de Man, République de Côte d'Ivoire. 27 p.

FAO 1994. FAO Investment Centre. Etude de l'impact de la dévaluation du FCFA sur la compétitivité des productions rizicoles dans les pays de l'UEMOA. Rapport $n^{\circ}$ 107/94 BOAD-WAF 2. Rome, Italie, $48 \mathrm{p}$.

Hirsch R. D., Inial J. L. et Y. Ficatier. 1984. La riziculture ivoirienne: Diagnostic et conditions préalables d'une relance. Côte d'Ivoire, $86 \mathrm{p}$

Koné D. 2003. Caractérisation du risque climatique de la culture de maïs en zone centre de Côte d'Ivoire. In Soil Water balance in the Sudano-Sahelian Zone. IAHS Publ N 199 pp 515 - 521.

Koné M. 1993. Analyse de l'impact de la vulgarisation agricole sur l'efficacité économique des paysans dans le nordouest et l'ouest de la Côte d'Ivoire, cas des départements de Touba et Biankouma, Thèse de Doctorat $3^{\text {ème }}$ cycle, CIRES, $172 \mathrm{p}$.

Léonard E. et M. Oswald. 1996. "Une agriculture forestière sans forêt. Transformation de l'environnement et mise en place de systèmes stables en Côte d'Ivoire forestière". Nature Science et Société, 1996 (4), $3 \mathrm{p}$

Martin F. 1988. "Food security and comparative advantage in Senegal : a Micro-Macro Approach." Ph.D. thesis, East Lansing, Mi. Michigan State University, 1988.

MINADER. 2002. Catalogue officiel des variétés de riz. Ministère de l'agriculture et du développement rural (MINADER), Côte d'Ivoire. Edition 2002, 44 p.

MINAGRA 1991. Note sur la libéralisation du marché intérieur du riz. DCGTX, Ministère de l'Agriculture et des Ressources Animales (MINAGRA), Document de travail, $11 \mathrm{p}$.

Poisson C. et S. Doumbia. 1987. Variétés nouvelles de riz. Conseils pratiques pour une riziculture moderne. Institut des Savanes (IDESSA), Centre des Cultures vivrières / Centre de Coopération Internationale en Recherche Agronomique pour le Développement, Département IRAT. Les Nouvelles Editions Africaines, $64 \mathrm{p}$.

Scandizzo P. L. and C. Bruce. 1980. Methodology for measuring agricultural price incentive effects. Washington DC : World Bank Staff Work. Pap. №. 344

Weber M. T., Staatz J. M., Holtzman J. H., Crawford E. W. and R. Bernsten R. 1988. Informing food security decisions in Africa: Empirical analysis and policy dialogue, American journal of Agricultural Economics, Vol. 70 (5) : 1044 - 1051. 